REVIEW ARTICLE

\title{
Is it Time to Review the Role of Continuous Glucose Monitoring Systems in Diabetes Management?
}

\author{
Le Minh Quang, MSc, $M D^{1,2^{*}}$ \\ ${ }^{1}$ Chief Operating Officer cum Outpatient Clinic and Services Director, City International Hospital Ho Chi \\ Minh City, Vietnam \\ ${ }^{2}$ Consultant Diabetologist, Cardiff Diabetes Centre \& Centre for Research and Control of Diabetes, Ho Chi \\ Minh City, Vietnam
}

*Corresponding author: Le Minh Quang, MSc, MD, Chief Operating Officer cum Outpatient Clinic and Services Director, City International Hospital and Consultant Diabetologist, Cardiff Diabetes Centre \& Centre for Research and Control of Diabetes - 763, Le Hong Phong Street, Ward 12, District 10, Ho Chi Minh City, Vietnam

\begin{abstract}
Key Messages
- The use of CGM are beneficial in glycemic control, reduction of hypoglycemia and hyperglycemia events, HbA1c reduction and improvement of quality of life in patients with diabetes.

- The barriers including the development of international guidelines on CGM use, the cost or reimbursement issues and the accuracy of CGM systems should be implemented for expanding the CGM use.
\end{abstract}

\begin{abstract}
Abbreviations and Acronyms
HbA1C: Hemoglobin A1c; CGM: Continuous Glucose Monitoring; $\mathrm{Cl}$ : Confidence Interval; CV: Cardiovascular; GV: Glucose Variability; SMBG: Self-Monitoring Blood Glucose; TIR: Time In Range; TIHyper: Time In Hyperglycemia; TIHypo: Time In Hypoglycemia; T1D: Type 1 Diabetes; T2D: Type 2 Diabetes

Continuous glucose monitoring systems help tracking patient glucose levels throughout 24 hours that provide a better glycemic management.
\end{abstract}

\section{Why we Need New Measures to Improve Dia- betes Management?}

Hemoglobin $\mathrm{A} 1 \mathrm{C}(\mathrm{HbA} 1 \mathrm{c})$ and self-monitoring of blood glucose (SMBG) are widely used as standardized measurements in diabetes management. $\mathrm{HbA1c}$ is a measure of the mean blood glucose level over a period of 8-12 weeks. This index is easy to measure, relatively inexpensive and internationally standardized. $\mathrm{HbA1c}$ helps to predict the complications, particularly micro- vascular complications in patients with diabetes [1]. However, because $\mathrm{HbA} 1 \mathrm{c}$ represents only an average measure of glucose levels, it does not provide glycemic variability (GV), glucose excursions or hypoglycemia. Hypoglycemia and GV ultimately are major challenges during optimization of glycemic control. GV is associated with an increased risk of adverse cardiovascular (CV) outcomes and diabetic retinopathy. There are many factors that affect GV including diet, physical activities, patient health status and therapeutic regimen. On the other hand, there are certain conditions and factors such as renal failure, anemia or hemoglobinopathies that can alter the accuracy of $\mathrm{HbA} 1 \mathrm{C}$ result $[2,3]$.

Current viewpoints in diabetes care emphasize personalized treatment plan for each individual to provide an excellent quality, to improve patient experience, and to minimize the burdens of daily living with diabetes. Many current guidelines indeed recommend an optimal glucose control consisting of an HbA1C level $\sim 7 \%$ (but personalized for each individual) with less GV and severe hypoglycemic events as much as possible [3].

SMBG for long is also an accurate measure of capillary glucose levels that is relatively inexpensive and easy to use. SMBG can improves blood glucose control. However, SMBG have many limitations such as representing only a single point value of glucose, no indica-

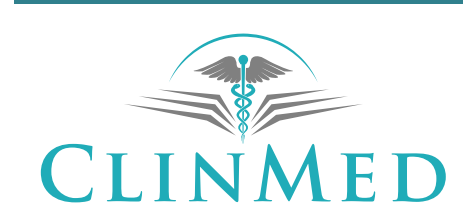

INTERNATIONAL LIBRARY 

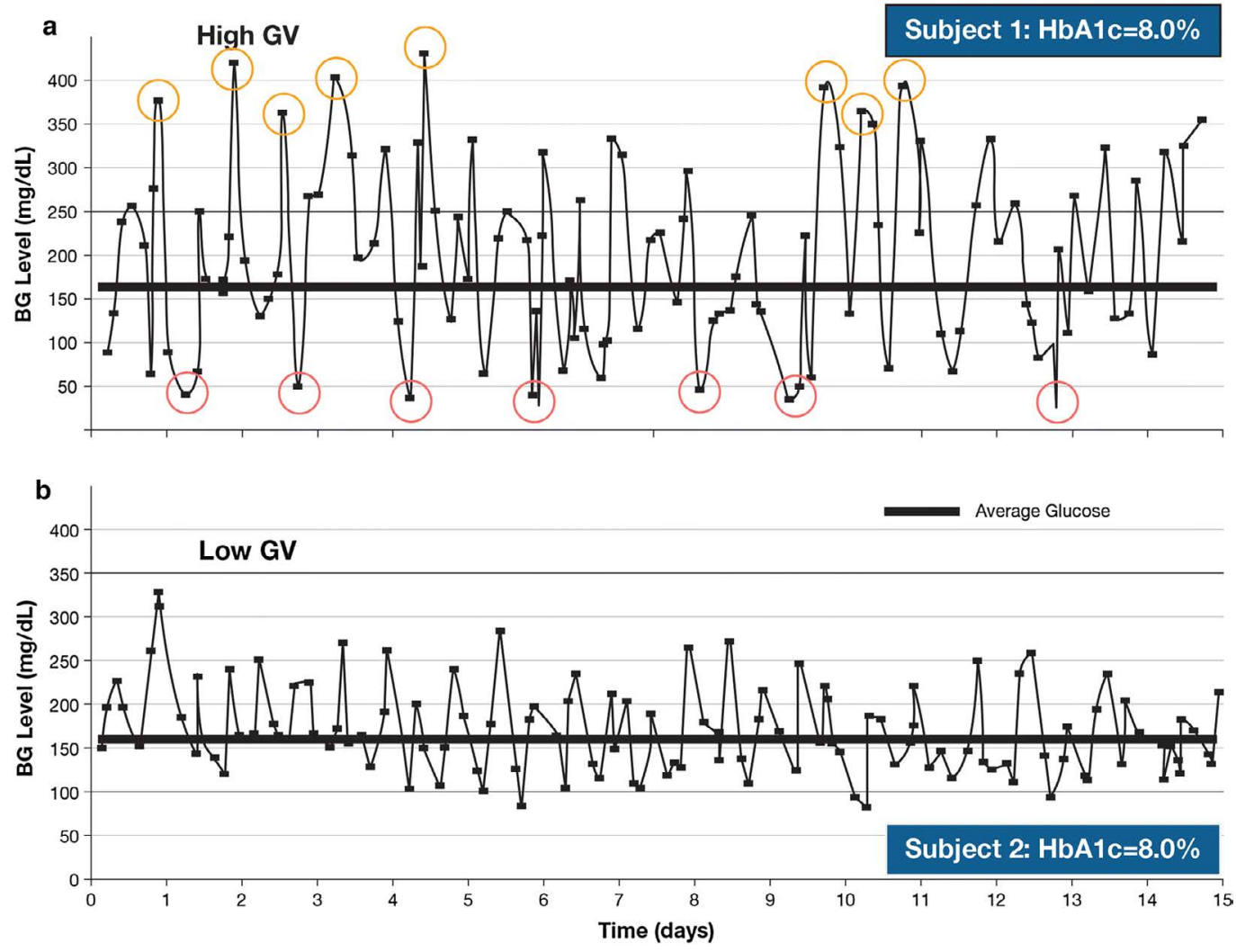

Figure 1: Differences in glycemic variability over 15 days in two patients with similar HbA1c levels. BG blood glucose, GV glycemic variability, HbA1c glycated hemoglobin A1c -Reproduced from Kovatchev and Cobelli [4].

tion of the trend or rate of glucose level change, multiple daily testing required that increase the daily burden and unable to detect nocturnal and unawareness hypoglycemic events (Figure 1).

\section{Benefits of Continuous Glucose Monitoring System}

The dramatically emergence of continuous glucose monitoring (CGM) systems has potentially become one of the disruptive innovations that change the way we manage the patients with diabetes. CGM systems are measured glucose readings in the interstitial fluid continuously throughout whole day. The glucose readings display in real-time manner including not only glucose levels but also the trends that can help patient and health care provider (HCP) make interventions before the events happen.

There are many research and analysis that show the benefits of CGM in patients with type 1 (T1D) and type 2 diabetes (T2D). Twenty-seven randomized controlled trials (RCTs) assessing the outcomes of CGM use in 3,826 patients have been published.

Compared to conventional $\mathrm{HbA1C}$ and SMBG measurements, CGM provides more valuable information including a continuous and real-time glucose monitoring, detection of GV and number and time of hypoglycemia as well as hyperglycemia. CGM can help to minimize severe or nocturnal hypoglycemia, especially in patients with hypoglycemic unawareness; CGM resulted in bet-
Table 1: The correlation of TIR and HbA1c. Reproduced from [7]

\begin{tabular}{|l|l|l|}
\hline Measured TIR (70-180 $\mathbf{~ m g / d L ) ~}$ & A1c & $\mathbf{9 5} \% \mathbf{C l}$ \\
\hline $40 \%$ & $8.1 \%$ & $7.1-9.1 \%$ \\
\hline $50 \%$ & $7.7 \%$ & $6.7-8.7 \%$ \\
\hline $60 \%$ & $7.3 \%$ & $6.3-8.3 \%$ \\
\hline $70 \%$ & $6.9 \%$ & $5.9-7.9 \%$ \\
\hline $80 \%$ & $6.5 \%$ & $5.5-7.5 \%$ \\
\hline
\end{tabular}

ter glycemic control than conventional treatment and reduce the mean amplitude of glycemic excursion in persons with diabetes. CGM reduces importantly $\mathrm{HbA1C}$ levels, increase time in range and reduce incidence and time spent with hypoglycemia. In addition, CGM can be used as a valuable tool for patient education of self-management and help patients to personalize their management strategies $[5,6]$.

\section{Current Recommendations on the CGM Use}

With the currently approved CGM systems available, it is practical and scalable to define an ideal approach of effectively diabetes management based on CGM data and reports with standardized visualizations. Many new definitions of glucose target values are developed and standardized such as time in range (TIR), time in hypoglycemia (TIHypo) or time in hyperglycemia (TIHyper) soon to become new goals in diabetes management along with conventional HbA1c. The more new glucose values fall in target range, the better the $\mathrm{HbA} 1 \mathrm{C}$ 
is likely to be because of its correlation (Table 1).

To achieve an optimal control, the TIR level should be maintained as high as possible while the TIHypo and TIHyper are kept at lowest levels. For instance, an $\mathrm{HbA} 1 \mathrm{c}$ is $6.9 \%$ is equal a TIR $(70-180 \mathrm{mg} / \mathrm{dL}) \sim 72 \%$ or 17.3 hours/day and TIHypo (> $180 \mathrm{mg} / \mathrm{dL}$ ) $\sim 25 \%$ or 6 hours/day $[8,9]$.

In fact, the recommendations of CGM use by professional bodies vary and are more consistent for patient with T1D than those with T2D. It is advisable to combine CGM alongside $\mathrm{HbA1c}$ monitoring to assess glycemic status and inform adjustments to therapy in all patients with T1D and in patients with T2D receiving intensive insulin therapy but out of control, especially in patients with recurrently severe hypoglycemia [10-13].

In a latest recommendation of the International Consensus, the panel recommends using a standardized CGM report and a 14-day composite glucose profile as a component in clinical decision-making. The ambulatory glucose profile (AGP) is considered as a standard CGM report. The TIR recommendations for most patients with T1D or T2D are as following:

- $70 \%$ of readings within a blood glucose range of 70$180 \mathrm{mg} / \mathrm{dL}(3.9-10.0 \mathrm{mmol} / \mathrm{L})$

- $<4 \%$ of readings $<70 \mathrm{mg} / \mathrm{dL}$ ( $<3.9 \mathrm{mmol} / \mathrm{L}$ )

- $<1 \%$ of readings $<54 \mathrm{mg} / \mathrm{dL}$ ( $<3.0 \mathrm{mmol} / \mathrm{L}$ )

- $<25 \%$ of readings $>180 \mathrm{mg} / \mathrm{dL}$ (> $10.0 \mathrm{mmol} / \mathrm{L}$ )
- $<5 \%$ of readings $>250 \mathrm{mg} / \mathrm{dL}$ ( $>13.9 \mathrm{mmol} / \mathrm{L}$ )

- For patients $<25$-years-old with an HbA1c goal is < $7.5 \%$, the TIR target should be set to about $60 \%$.

- For medically fragile patients, $>50 \%$ of TIR is a reasonable target. The time below range $(<70 \mathrm{mg} / \mathrm{dL})$ should be kept $<1 \%$ in this population.

- For pregnant patients with T1D, $>70 \%$ of CGM readings should be maintained within the range of 63 to $140 \mathrm{mg} / \mathrm{dL}$.

- For pregnant patients with T2D or gestational diabetes, $>90 \%$ of CGM readings should be maintained within the range of 63 to $140 \mathrm{mg} / \mathrm{dL}$ [14] (Figure 2).

\section{Conclusion}

Evidence from the studies and meta-analysis have shown that the application of CGM in patients with diabetes has been beneficial in glycemic control, reduction of hypoglycemia and hyperglycemia events, $\mathrm{HbA1c}$ reduction and improvement of quality of life. CGM use in combination of HbA1c monitoring obviously help to achieve a better optimal and stable glycemic control as well as to build patient confidence in self-management with a useful monitoring tool. While the use of CGM is drastically increasing, there are barriers including the absence of international guidelines on CGM use, the cost or reimbursement issues, frustration over adherence, the complexity of technology and the lack of accuracy needed to have proper solutions for enhancing routine use of CGM in patients with diabetes.
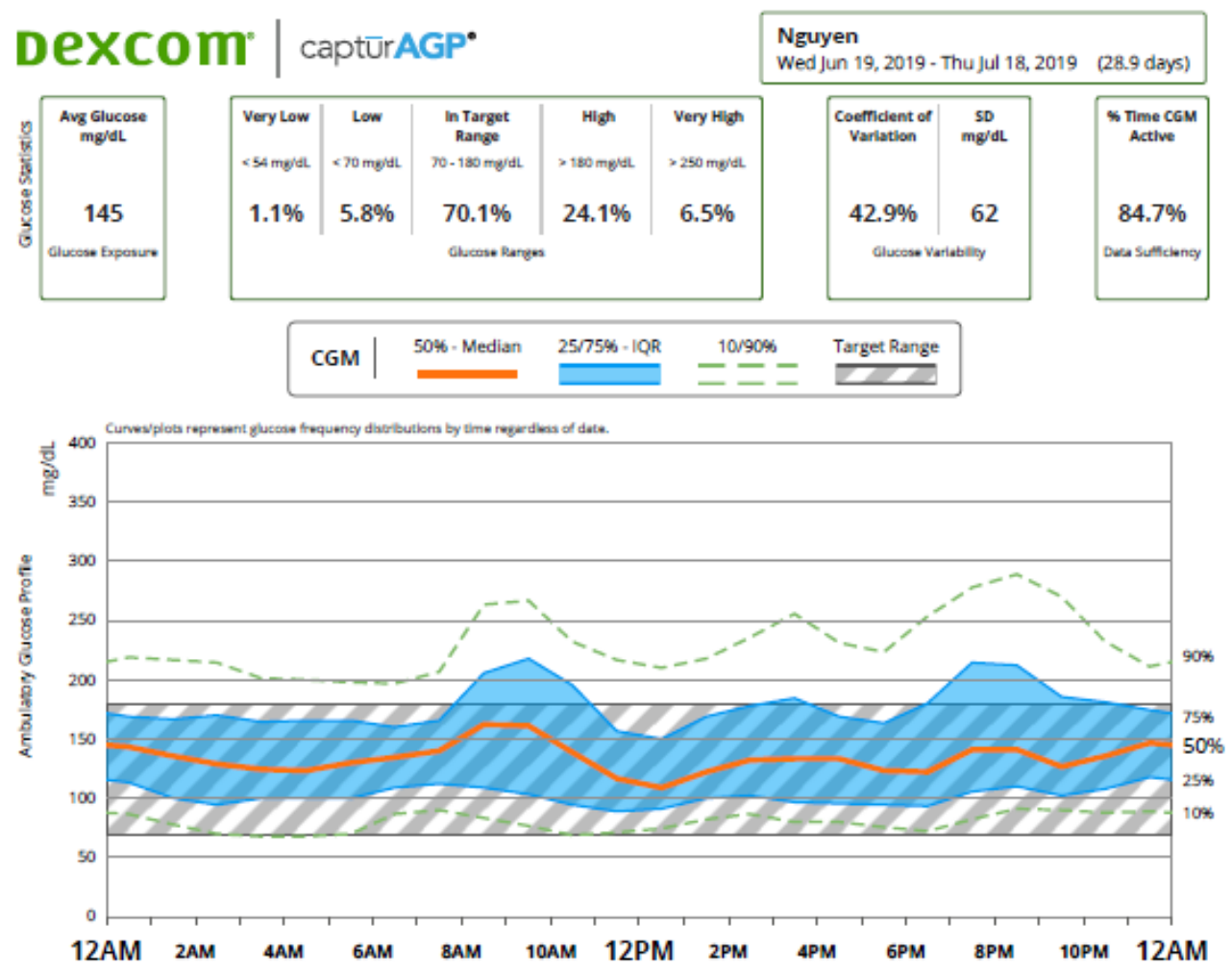

Figure 2: An Ambulatory Glucose Profile. 


\section{Conflicts of Interests}

The author declares no support from any organization for the submitted work.

\section{References}

1. UK Prospective Diabetes Study (UKPDS) Group (1998) Intensive blood-glucose control with sulphonylureas or insulin compared with conventional treatment and risk of complications in patients with type 2 diabetes (UKPDS 33). Lancet 352: 837-853.

2. Rubinow KB, Hirsch IB (2011) Reexamining metrics for glucose control. JAMA 305: 1132-1133.

3. Cryer PE (2014) Glycemic goals in diabetes: Trade-off between glycemic control and iatrogenic hypoglycemia. Diabetes 63: 2188-2195.

4. Kovatchev B, Cobelli C (2016) Glucose variability: Timing, risk analysis, and relationship to hypoglycemia in diabetes. Diabetes Care 39: 502-510.

5. Hirsch IB, Battelino T, Peters AL, Chamberlain JJ, Aleppo $G$, et al. (2018) Role of continuous glucose monitoring in diabetes treatment. Arlington (VA): American Diabetes Association.

6. Ajjan R, Slattery D, Wright E (2019) Continuous glucose monitoring: A brief review for primary care practitioners. Adv Ther 36: 579-596.

7. Beck RW, Connor CG, Mullen DM, Wesley DM, Bergenstal RM (2017) The fallacy of average: How using HbA1c alone to assess glycemic control can be misleading. Diabetes Care 40: 994-999.

8. Bergenstal RM (2015) Glycemic variability and diabetes complications: Does it matter? Simply put, there are better glycemic markers. Diabetes Care 38: 1615-1621.

9. Bergenstal RM, Garg S, Weinzimer SA, Buckingham BA, Bode BW, et al. (2016) Safety of a hybrid closed-loop insulin delivery system in patients with type 1 diabetes. JAMA 316: 1407-1408.

10. American Diabetes Association (2018) Standards of medical care in diabetes-2018. Diabetes Care 41: S1-153.

11. Danne T, Nimri R, Battelino T, Bergenstal RM, Close KL, et al. (2017) International consensus on use of continuous glucose monitoring. Diabetes Care 40: 1631-1640.

12. Bailey TS, Grunberger G, Bode BW, Handelsman Y, Hirsch IB, et al. (2016) American Association of Clinical Endocrinologists and American College of Endocrinology 2016 outpatient glucose monitoring consensus statement. Endocr Pract 22: 231-261.

13. Peters AL, Ahmann AJ, Battelino T, Evert A, Hirsch IB, et al. (2016) Diabetes technology-continuous subcutaneous insulin infusion therapy and continuous glucose monitoring in adults: An endocrine society clinical practice guideline. $J$ Clin Endocrinol Metab 101: 3922-3937.

14. Battelino T, Danne T, Bergenstal RM, Amiel SA, Beck $R$, et al. (2019) Clinical targets for continuous glucose monitoring data interpretation: Recommendations from the international consensus on time in range. Diabetes care 42: 1593-1603. 